\title{
Modeling, Simulation and Development of a Model Predictive Controller for an Autonomous Steer by Wire ADS-DV
}

\author{
${ }^{1}$ Mohamed Abdlshakour Allam, ${ }^{2}$ M.Ibrahim Abdelziz, ${ }^{3}$ Diaa Abidou \\ ${ }^{1,2,3}$ Department of Automotive Engineering, Faculty of Engineering, Ain Shams University, Cairo, Egypt 11517
}

\begin{abstract}
An autonomous vehicle can maneuver its routes in shorter time if it can navigate on the limits of handling. This requires accurate steering commands to be applied to the vehicle which can be generated through an accurate steer by wire system that it's properly modeled along with the vehicle dynamics. As well as a robust controller that can handle the different driving conditions of the Autonomous vehicle. A mathematical model for autonomous vehicle dynamics is proposed to make use of the maximum attainable acceleration figures the tire can handle without losing traction. As well as investigating the vehicle stability during maneuvers proposed by the higherlevel control modules. The model integrates a Steer by Wire System with a simplified electric power train as well as the handling dynamics of the vehicle. It takes into consideration forward, lateral, yaw and roll motions which is more exact and effective than simple bicycle planar models. The stability of the vehicle is investigated as well as its maneuverability. The handling limits of the vehicle are simulated using the g-g diagram under different maneuvers. Lateral and longitudinal accelerations, lateral velocity, slip angle, side forces and yaw motion are all investigated as well as the total acceleration which is plotted on a g-g diagram.
\end{abstract}

A Model Predictive Controller (MPC) for the Steer by wire system is developed to accommodate all the navigation conditions of the vehicle, changes in loading and commands provided by the perception and path planning system. The controller follows the commands given by these systems and provides the recommended maneuver with respect to the vehicle handling limits bounded by the g-g diagram. The controller optimizes the path decided by the decision-making systems and provides the fastest and most stable maneuver without losing control like skidding or roll over.

Keywords: Model Predictive Control, Autonomous Vehicles, Handling Dynamics, g-g diagram, lateral acceleration, MATLAB Simulink, Bicycle Model, Steer by wire, Maneuverability, Roll motion, Control at the limit.

\section{INTRODUCTION}

Several publications investigated handling dynamics at the limit: Yung-Hsiang et al. [5] used a model-based estimation method utilizing pneumatic trail data to identify the handling limits taking advantage of early friction information in the tire's pneumatic trail. Nakajima et al. [6] and Yasui et al. [7] used the concept of self-aligning torque (AT) to predict the vehicle's handling limit where maximum AT occurs before maximum cornering forces are reached without depending on yaw rate data. Ono et al. [8] used an estimation method of the tire grip margin. A brush model evaluates its robustness by applying the method to a magic formula tire model, which adapts to the changes of the tire conditions.

Other publications studied autonomous vehicles at the limits of handling where these efforts relied on the kinematic bicycle model for vehicle simulation at the friction limits while considering the steering system as a fixed gain which introduces undesirable errors especially at the friction limits.: Borrelli et al. [1] and Keviczky et al. [2] used a model predictive control approach relying on the bicycle model for vehicle dynamics modeling. Nitin et al [3] designed a lane keeping system for path tracking and stability at the limits also using the bicycle model and Kapania et al. [4] used the same model to design a path tracking controller via iterative learning control. Funke et al. [5] implemented a collision avoidance and stabilization system for autonomous systems in emergency scenarios while relying on the same concept.

Polack et al. [6] compares the validity of the linear bicycle model with a more complicated 9 degree of freedom model. Where in their work, it was demonstrated that the bicycle model loses its consistency at lateral accelerations above $0.5 \mu \mathrm{g} \mathrm{m} / \mathrm{s}^{2}$. The inconsistency becomes predominant when the limitations on lateral acceleration are neglected. This is due to the effect of rolling and high lateral weight transfer between tires at such high acceleration figures.

Our research uses four degrees of freedom model combined with the steering system model as well as a longitudinal forward dynamics one to plot the total 
acceleration of the vehicle on the g-g diagram. The target is to develop an analytic model which is realistic and at the same time computationally efficient to be integrated in a model predictive controller (MPC) for the autonomous golf cart where a simple linear tire model was used and errors due to the tire nonlinearities are compensated by providing the controller with steering and position feedback.

Model predictive controls in the automotive field depend on the hypothesis that control problems in automotive systems can be outlined using an optimal multi input multi output (MIMO) problem [7]. Where in many cases the problem can be defined using the system states which change according to several inputs and are controlled by some variables. These variables are limited to a certain range and behavior. The system response is also physically bounded to several factors. The automotive control problem proposed in the control design in this work is the vehicle stability and path following. Where the ADS-DV (Autonomous Driving System Dedicated Vehicle) is required to navigate the routes of the faculty of engineering premises using sensor data. The sensors propose a certain path and vehicle speed and is required to follow these commands as closely as possible in order to stay within the required trajectory.

MPC is especially efficient in MIMO systems where the inputs and outputs interact heavily and are greatly dependable where the use of Proportional Integral Derivative (PID) controllers proves to be a challenging design process where the inputs are controlled independently for each control loop. MPC controls all the variables while considering all the system variables and interactions. MPC also is highly efficient in tracking constraints set by the physical limits of the plant or even other constraints set by external disturbances. Another benefit of MPC is its ability to predict the future using the "prediction model" referred to as "preview capability". It can easily incorporate future reference information into the control problem to improve the controller performance. To summarize, MPC uses the model of the studied system in order to predict its behavior in the future, it solves an online optimization problem selecting the optimum control action that drives the predicted output value to the reference value. All this is done with respect to the MIMOs, physical constraints and measured disturbances.

The basic idea of MPC is the optimization of the future behavior of the studied system using a mathematical model over a finite time horizon which is generally referred to as the "prediction horizon"[8]. The optimization is done at each sampling instant $\mathrm{n}$ where the resulting element of the optimal control sequence is used as a feedback control value for the next step. The control action is optimized based on an objective function which is also referred to as the "cost function" where the optimum control action "manipulated variable" is the one that minimizes the cost function. The general concept of MPC is illustrated in Figure 1.

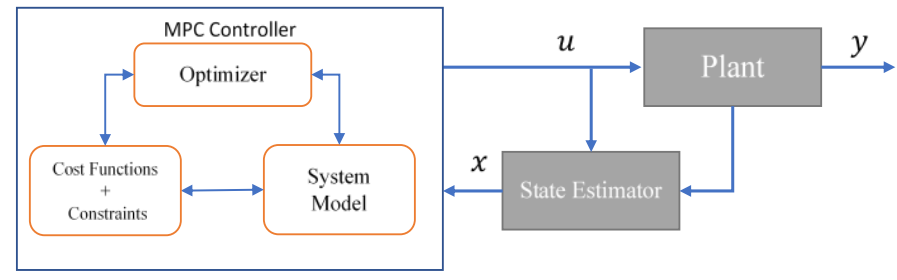

Figure 1: MPC Concept

An example for MPC is a vehicle required to change lanes from the right lane to the middle lane using only the steering command while the longitudinal velocity is kept constant shown in Figure 2 (a) [7]. The middle lane in grey will be the input reference to the MPC using the lateral position as feedback where the MPC uses the plant or vehicle model at the current time " $t$ " to simulate the vehicle path "lateral position" for the next time step " $\mathrm{t}+1$ " if the steering wheel was turned at trail increment shown in Figure 2 (b).

"P" or the "prediction horizon" indicates how far into the future the MPC investigates which is often represented by the length of time in the future or the time steps to simulate in the future. The controller simulates several inputs to the plant model which correspond to different lateral positions shown in Figure 2 (c), (d), (e). The paths are represented by black, orange and green lines. This is done to find the optimum steering command required to follow the required reference as closely as possible. The optimization process is done in an organized systematic way using an optimizer which solves an online optimization problem. By solving the online optimization problem. The MPC finds the best steering command which minimizes the error between the predicted path and the reference path.

The controller also follows the constraints set by the physical limits of the car such as the tire road interactions or occupants comfort controlled by the rate of change of the steering wheel angle and any other constraints set. The online optimization problem minimizes a "cost function" including the weighted sum of all the predicted errors and the steering wheel angle increments shown in Figure $2(\mathrm{~g})$. The cost function " $\mathrm{J}$ " is as follows:

$$
\text { Cost function }=J=\sum_{i=1}^{t} w_{e} e_{k+1}^{2}+\sum_{i=0}^{t-1} w_{\Delta u} \Delta u_{k+i}^{2}
$$

The MPC solves the online optimization problem at the current time step over the selected prediction horizon all while following the set constraints. The path output by the 
ISSN (online): 2581-3048

Volume 4, Issue 5, pp 24-33, May-2020

https://doi.org/10.47001/IRJIET/2020.405005

optimization problem which yields the smallest cost function is the optimal solution or the best steering angle. MPC only applies the steering command at the current time step of the sequence of the prediction horizon and the rest are disregarded. The MPC then uses the feedback from the measured lateral position to determine the error and starts solving the online optimization problem for the new time step. The measured feedback maybe different from the predicted one by the MPC due to external disturbances or other factors. As the MPC moves to the next time increment, the prediction horizon shifts forwards as shown in Figure 2 (f) and the calculation is redone to determine the new optimal steering wheel angle.

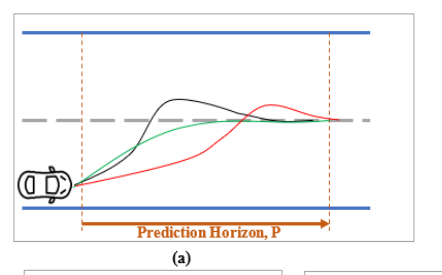

(a)

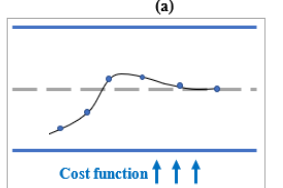

(c)

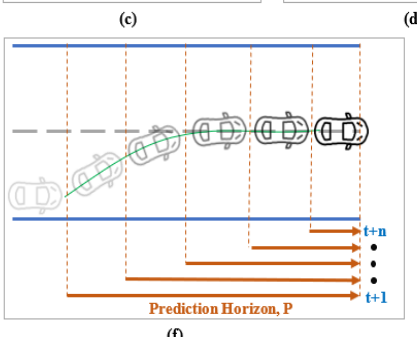

(d)

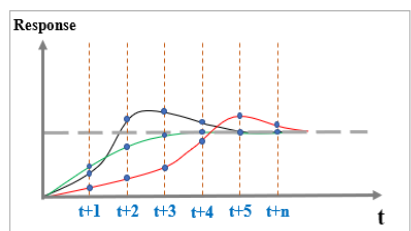

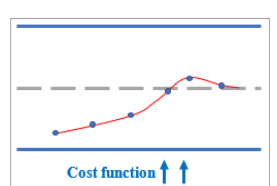
d)

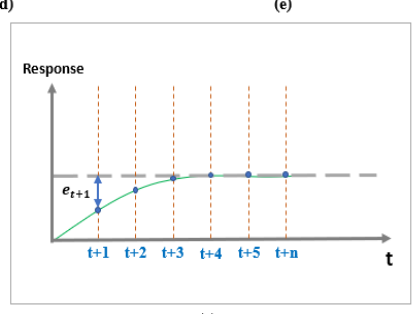

(g)
Figure 2: Concept for model predictive control for lane change with tracking

The controller design parameters are also a factor in the optimization problem solution. The main design parameters affecting the solution are the sample time, prediction horizon, control horizon, constraints and constraints weights.

The sample time indicates the rate by which the controller executes the control algorithms, choosing a sample time that is too big makes yields a longer reaction time which is inefficient in handling disturbances, while a small sample time will add to the computational load for the controller to operate. Selecting the proper sample time strikes balance between controller robustness and computational load.

As shown in Figure 3 (a), (b); the prediction horizon indicates how far the controller predicts into the future and must be optimized since choosing a small horizon can make the controller too slow and will react to the predicted system dynamics too late. Choosing a longer prediction horizon will introduce the possibility of a larger computational load without benefit due to the change of the system dynamics which is a computational waste illustrated in brown Figure 3 (a), (b). Another parameter of the MPC design is the control horizon which maybe illustrated by assuming that the set of control actions leads to the predicted output, then the control horizon is the control actions to the final time step " $n$ ". The rest of the control actions are held constant. Setting the control horizon at a smaller value decreases the computational load but at the same time may not give the optimum maneuver over the prediction horizon. This is due to the changing system dynamics in the next time steps. By maximizing the control horizon an optimized maneuver is reached however on the cost of model complexity where normally the first few control actions have an effect on the system output which is also shown in Figure 3 (a), (b) in blue.
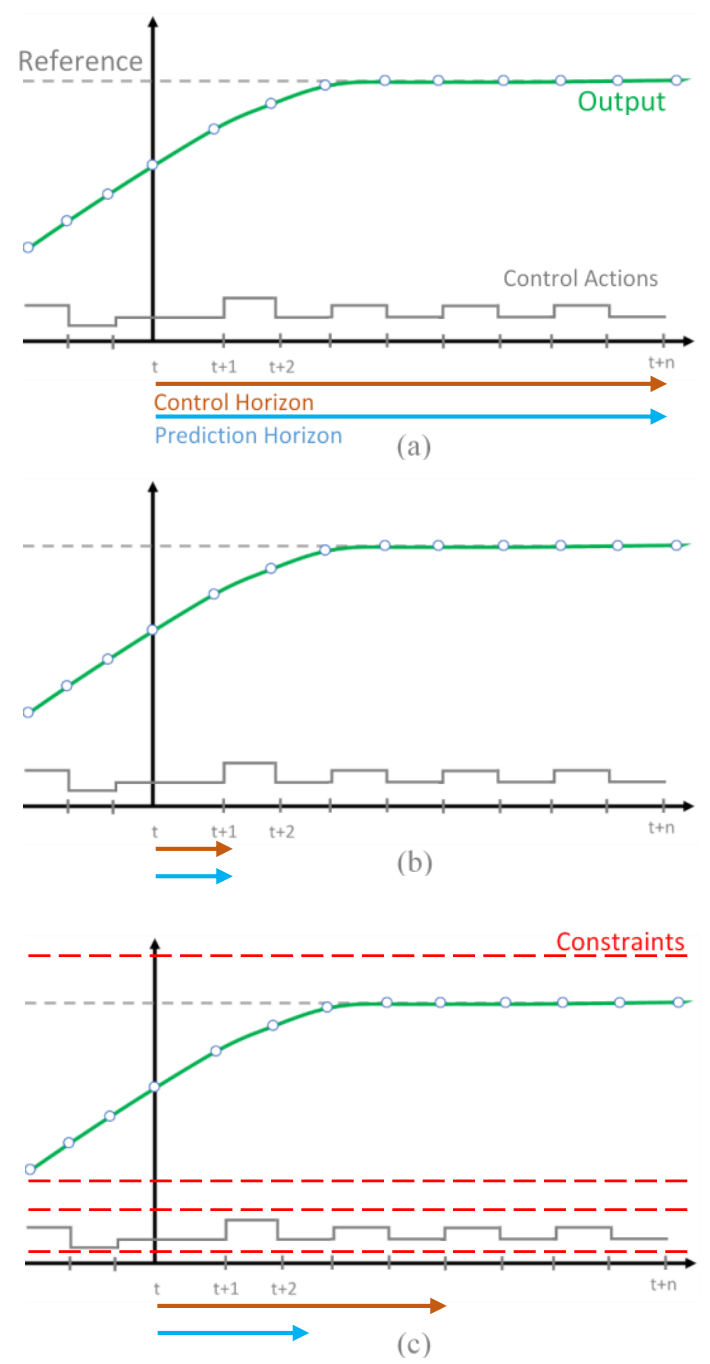

Figure 3: MPC design parameters

MPC can adhere to constraints of the physical system which can be set on the inputs and output and their rate of change. They may be set as soft or hard constraints where hard controls are followed strictly by the controller and cannot be 
violated. On the other hand, soft constraints can be violated depending on the output response of the vehicle.

Setting too many constraints may lead to an infeasible solution and would lead to controller failure and the optimization problem is unsolvable. The overall design parameters of MPC are illustrated in Figure 3.

\section{VEHICLE DYNAMICS PREDICTION MODEL}

The control problem in this research is for dynamic stability bounded by the vehicle response in terms of total acceleration, yaw velocity, vehicle position, yaw and slip angles. The maximum total acceleration is limited by the tireroad interactions as well as the maximum attainable side slip angle. The vehicle position is also bounded by the proposed path set by the path planning system. The variable to be manipulated also has limitations. For example, the rate of steering angle change is limited by the electric motor maximum available rotational speed. Also, the maximum steering angle is also limited to the steering system mechanical design and the vehicle tires.

The control problem stated here is a multi-objective one where several conflicting variables need to optimized using a limited range of inputs and outputs. Usually in MPC one variable is chosen to be optimized whether the value should be strictly followed, minimized or maximized. While the other variables are set as constraints.

In the optimization problem the controlled variable is the vehicle steering angle. The limitations are from the vehicle required path, where the controller must manipulate the steering angle in order to follow the exact proposed position while at the same time adhering to the limitations of the maximum available steering angle, rate of change of steering angle, maximum available total acceleration, maximum yaw angle and yaw rate for the comfort of the driver.

The optimal problem solution works at follows; the path planning algorithm sets a certain trajectory in terms of vehicle position, yaw rate and longitudinal speed. Then the controller uses the initial values to calculate the values of the steering angle in the next time steps in order to minimize the difference between the requested position and yaw angle and the actual one. The total acceleration is kept below the set value as well as following the restrictions on the steering angle rate and limitations.

The prediction model for the vehicle handling dynamics is the modified 4DOF double track model which is coupled with a longitudinal power train and steering kinematics model is shown in Figure 4 [9].

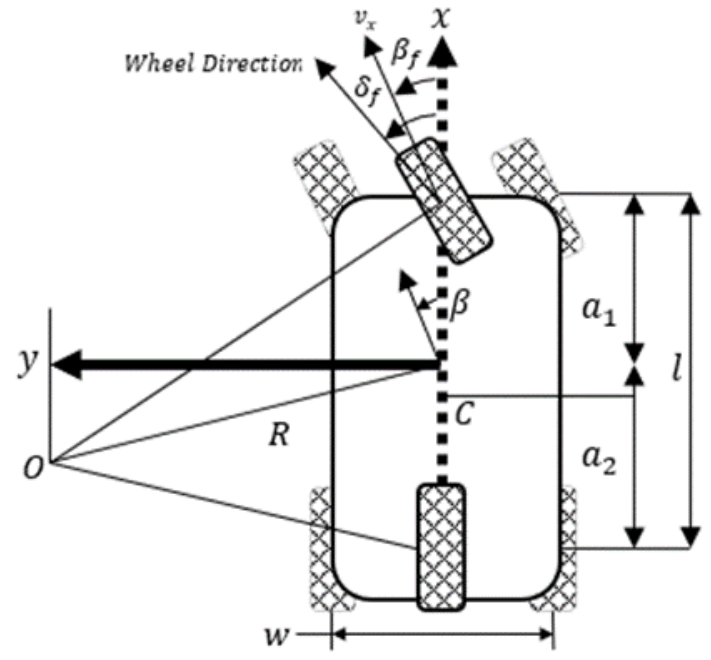

Figure 4: Dynamic vehicle model

The steer angle $\delta$ is assumed to be the manipulated variable and the other variables $v_{y}, \rho, \varphi, r$ are assumed as the output, the control equation representing the system can be written as:

$$
\dot{q}=[A] q+u
$$

Where the output matrix $q^{\circ}$ can be described as:

$$
q=\left[\begin{array}{l}
v_{y} \\
p \\
\varphi \\
r
\end{array}\right]
$$

And $[\mathrm{A}]$ is the coefficient matrix denoted by:

$$
[A]=\left[\begin{array}{cccc}
\frac{C_{\beta}}{m v_{x}} & \frac{C_{p}}{m} & \frac{C_{\varphi}}{m} & \frac{C_{r}}{m}-v_{x} \\
\frac{E_{\beta}}{I_{x} v_{x}} & \frac{E_{p}}{I_{x}} & \frac{E_{\varphi}}{I_{x}} & \frac{E_{r}}{I_{x}} \\
0 & \mathbf{1} & 0 & 0 \\
\frac{D_{\beta}}{I_{z} v_{x}} & \frac{D_{p}}{I_{z}} & \frac{D_{\varphi}}{I_{z}} & \frac{D_{r}}{I_{z}}
\end{array}\right]
$$

$\mathrm{q}$ is the control variables vector calculated from:

$$
q=\left[\begin{array}{l}
v_{y} \\
p \\
\varphi \\
r
\end{array}\right]
$$

Finally, $\mathrm{u}$ is the input vector where:

$$
\boldsymbol{u}=\left[\begin{array}{c}
\frac{C_{\delta}}{\boldsymbol{m}} \\
\frac{E_{\delta}}{I_{x}} \\
\mathbf{0} \\
\frac{D_{\delta}}{I_{z}}
\end{array}\right] \delta
$$

The longitudinal acceleration of the body frame derived from the longitudinal dynamics model can be described as: 
ISSN (online): 2581-3048

$$
a_{x}=\frac{1}{m} \sum F_{x}+r v_{y}
$$

While the lateral acceleration in the body frame is derived from the lateral dynamics model can be calculated as:

$$
a_{y}=r v_{x}+v_{y}
$$

The vehicle total acceleration is bounded by the measured maximum deceleration which was $0.6 \mathrm{~g}$ where it can be stated as [10]:

$$
a_{t}=\sqrt{a_{x}^{2}+a_{y}^{2}}
$$

Where:

$$
\mu g \geq \sqrt{a_{x}^{2}+a_{y}^{2}}, t \geq 0
$$

or

$$
\mu g \geq \sqrt{\left(\frac{1}{m} \sum F_{x}+r v_{y}\right)^{2}+\left(r v_{x}+\dot{v}_{y}\right)}, t \geq 0
$$

The condition for total acceleration is derived from the $\mathrm{g}$ $\mathrm{g}$ diagram where the lateral acceleration is plotted on the horizontal axis and the longitudinal acceleration is plotted on the vertical axis.

The MPC discretizes the model and the state equation into sampling time " $\mathrm{t}$ " in order to formulate and solve the online optimization problem and minimize the cost function " J". At each sampling time a desired precomputed trajectory over the "prediction horizon" of " $\mathrm{n}$ " steps where $\mathrm{n} \in \mathbf{Z}^{+}$. This is given in a sequence of lateral and longitudinal positions $\left(\boldsymbol{X}_{\boldsymbol{i}}^{r}, \boldsymbol{Y}_{\boldsymbol{i}}^{\boldsymbol{r}}\right)$, Where $\boldsymbol{i}=\boldsymbol{t}+\mathbf{1}, \ldots, \boldsymbol{t}+\boldsymbol{n}$. Where the cost function can be described as:

$$
\begin{aligned}
& J\left(A_{x_{t}},\right.\left.A_{t_{t}}, X_{t}, Y_{t}, \Gamma_{t}, v_{d e s}, \delta_{f}, X_{r}, Y_{r}, \Gamma_{r}\right) \\
&= \sum_{i=t+1}^{t+n} \alpha_{1}\left[\left(X_{i}-X_{i}^{r}\right)^{2}+\left(Y_{i}-Y_{i}^{r}\right)^{2}\right. \\
&+\sum_{i=t}^{t+n-1}\left[\alpha_{2}\left(v_{x_{i, t}}-v_{d e s}\right)^{2}+\alpha_{3} a_{x_{i, t}}^{2}\right] \\
&+\sum_{i=t}^{t+n-1}\left[\alpha_{4}\left(\Gamma_{i, t}-\Gamma_{i}^{r}\right)^{2}+\alpha_{5} a_{t_{i, t}}^{2}\right] \\
& A_{x_{t}}=\left[a_{x_{t}}\right]
\end{aligned}
$$

Where, $\boldsymbol{A}_{x_{t}}=\left[\boldsymbol{a}_{x_{t, t}, \ldots,} \boldsymbol{a}_{x_{t, t+n-1}}\right], \boldsymbol{A}_{t_{t}}=\left[\boldsymbol{a}_{t_{t, t}}, \ldots, \boldsymbol{a}_{t_{t, t+n-1}}\right]$,

$X_{t}=\left[X_{t+1}, \ldots, X_{t+n}\right], Y_{t}=\left[Y_{t+1}, \ldots, Y_{t+n}\right]$,

$\Gamma_{t}=\left[\Gamma_{t+1}, \ldots, \Gamma_{t+n}\right], X_{t}^{r}=\left[X_{t+1}^{r}, \ldots, X_{t+n}^{r}\right], Y_{t}^{r}$

$$
=\left[Y_{t+1}^{r}, \ldots, Y_{t+n}^{r}\right] \text {. }
$$

In the cost function each sum represents a penalty over the deviation from a set point, where the first sum is for the deviation from the lateral or longitudinal position, the second sum is for the deviation from the desired forward velocity, while the final sum represents the deviation from the required yaw angle and the constraint over the total acceleration. The sums are weighed using the tuning parameters of $\boldsymbol{\alpha}_{1, \ldots, 5}$ at each time $\boldsymbol{t}=\boldsymbol{k} \boldsymbol{T} \boldsymbol{n}_{\boldsymbol{t}}$, with $\boldsymbol{n}_{\boldsymbol{t}} \in \boldsymbol{Z}^{+}, \boldsymbol{n} \geq \boldsymbol{n}_{\boldsymbol{t}}$ and $\boldsymbol{k} \in \boldsymbol{Z}^{+}$. The optimization problem to solved is:

$$
\min _{A_{t}, X_{t}, Y_{t} \Gamma_{t}} J\left(A_{x_{t}}, A_{t_{t}}, X_{t}, Y_{t}, \Gamma_{t}, v_{d e s}, \delta_{f}, X_{r}, Y_{r}, \Gamma_{r}\right),
$$

Which is subject to the conditions:

$$
\begin{gathered}
\mu g \geq \sqrt{\left(\frac{1}{m} \sum F_{x, k, t}+r_{k, t} v_{k, y, t}\right)^{2}+\left(r_{k, t} v_{k, x, t}+v_{k, y, t}\right)} \\
\delta_{f_{\min } \leq \delta_{f} \leq \delta_{f_{\max }}} \\
\delta_{\dot{f}_{\min } \leq \delta_{f} \leq \delta_{f_{\max }}^{\dot{m}_{\max }}} \\
k=t, \ldots, t+n
\end{gathered}
$$

Once a solution to minimize the cost function $\boldsymbol{J}$ is reached, the first samples of longitudinal and lateral position, yaw rate and total acceleration are provided as feedback to the controller and are used as a reference trajectory at a time of $\boldsymbol{t}+\boldsymbol{n} \boldsymbol{T}$. The cost function $\boldsymbol{J}$ is then solved over a shifted prediction horizon based on the feedback from the previous time step.

\section{SIMULATION SCENARIOS DESCRIPTION}

The designed MPC proposed was validated by simulation using the MATLAB driving scenario designer as sensor data due to the lack of availability of actual sensor data where the driving scenario designer provides a synthetic driving scenario for autonomous testing and validation.

The scenario designer is able to create roads, actors (pedestrians, cones, barriers ... etc.), lanes, vehicles and road markings. It is also capable of providing the vehicle position, velocity, yaw angle and rate and lateral and longitudinal accelerations. These signals can be the used as reference points for the designed MPC to follow instead of actual data from sensors to validate the controller.

The simulation scenarios included two different tests which are a moose test and a lane change maneuver. Tests were done at speeds which will introduce spikes in the vehicle total acceleration in order to induce the controller to enforce the constraints provided by the vehicle physical limitations.

The objective of all scenarios simulated is to ensure the vehicle stays within the stability envelope of total acceleration while adhering to the limitations on lateral position, maximum steering angle, and maximum steering angle rate and minimize the deviation from the proposed yaw angle. The moose test was chosen as it introduces high lateral acceleration in several 
directions in a short span of time which is perfect for the proposed controller algorithm.

\section{a) Moose Test}

The moose test is an important measure of vehicle handling performance, as it introduces behavior that is close to the vehicle handling limits which is convenient to test the designed MPC so close to the vehicle stability envelope. The standard dimensions of the "Moose test" or as sometimes referred to as the "Double lane change" (DLC) maneuver is shown in Figure 5 [11].
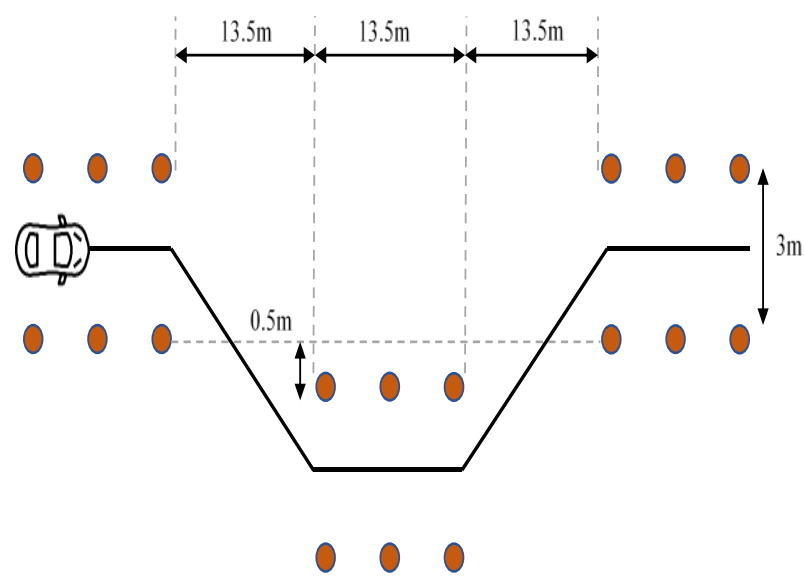

Figure 5: German Alliance of Automotive industry (VDA) "Moose Test" ISO 3888-2

The test consists of several steps as shown in Figure 6 obtained from the driving scenario designer, the vehicle is first accelerated to the desired speed which is normally around $90 \mathrm{~km} / \mathrm{hr}$ for a regular vehicle. For the vehicle of this study the vehicle speed is simulated between at $20 \mathrm{~km} / \mathrm{hr}$ as the maximum speed is $40 \mathrm{~km} / \mathrm{hr}$.

The vehicle is required to navigate at a speed which maximizes the tire forces given by the total available acceleration. As the vehicle enters the corner the throttle is held steady, and the steering commands are applied in order to change the lane twice.

The controller obtains the required steering angle in order to follow the trajectory as closely as possible. No braking or acceleration torques are applied here. However, the speed may decrease as the maneuver proceeds.
https://doi.org/10.47001/IRJIET/2020.405005

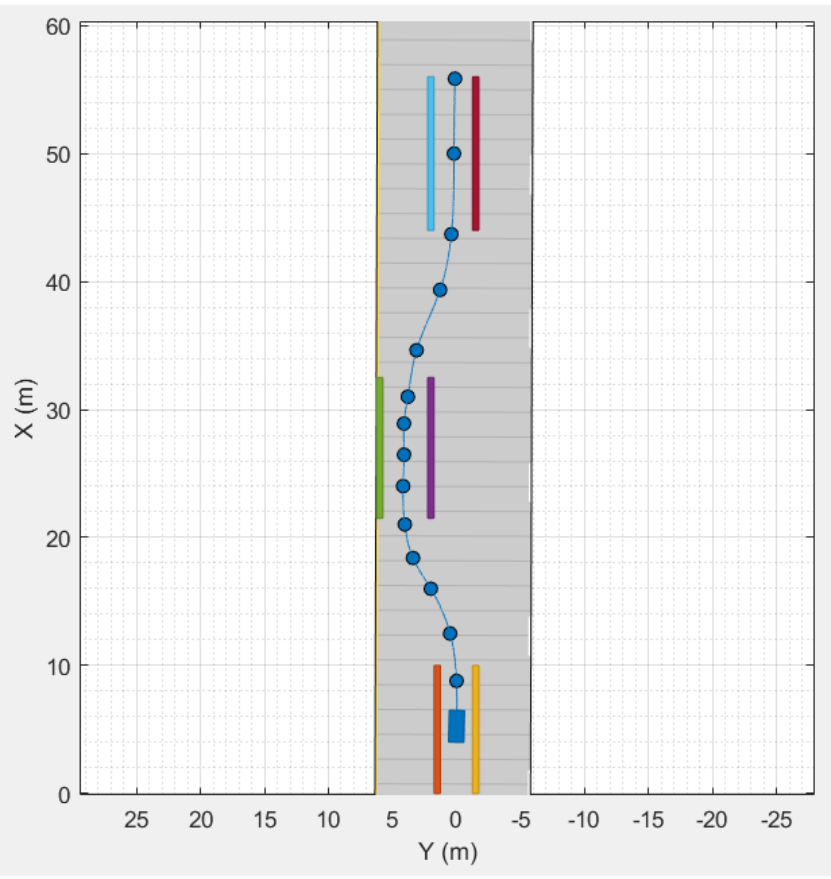

Figure 6: Moose test using the driving scenario designer

In this test the entry speed of the vehicle is set at $\mathbf{2 0} \mathbf{~ k m} /$ hr. the simulation results are shown in Figure 7 to 11 . The controller still shows acceptable reference tracking at increased entry speed presented in Figure 7 (a), (b) where the maximum deviation from the reference lateral position to be followed is around $0.31 \mathrm{~m}$ at $\mathbf{t}=\mathbf{1 . 8 s}$. The tracking error is accepted given that the vehicle reference total acceleration is more than $60 \%$ or $3.5 \mathrm{~m} / \mathrm{s}^{2}$ above the set maximum acceleration of $\mathbf{5 . 8 8 6 m} / \mathbf{s}^{2}$ or $0.6 \mathrm{~g} \mathrm{~m} / \mathbf{s}^{2}$ presented in Figure 7 (e), (f).

Figure 7 (f) Also shows the deviation of the vehicle total acceleration from the maximum set value indicating that the reference trajectory of the vehicle makes it lose control three times during the maneuver. First at $\mathbf{t}=\mathbf{1 . 8 s}$ with an acceleration deviation of $\mathbf{3 . 5 m} / \mathrm{s}^{2}$ giving the maximum value for lateral position tracking error. Then at $\mathbf{t}=\mathbf{2 . 4 5}$ with deviation of $\mathbf{1 . 2} \mathrm{m} / \mathrm{s}^{2}$ and a tracking error $\mathbf{0 . 1 m}$. Finally at $\mathbf{t}=\mathbf{8 . 2 \mathrm { s }}$ and deviation of around $\mathbf{2} \mathrm{m} / \mathrm{s}^{2}$ where the tracking error is less than $\mathbf{0 . 2} \mathbf{~ m}$. 
ISSN (online): 2581-3048

IRJIET
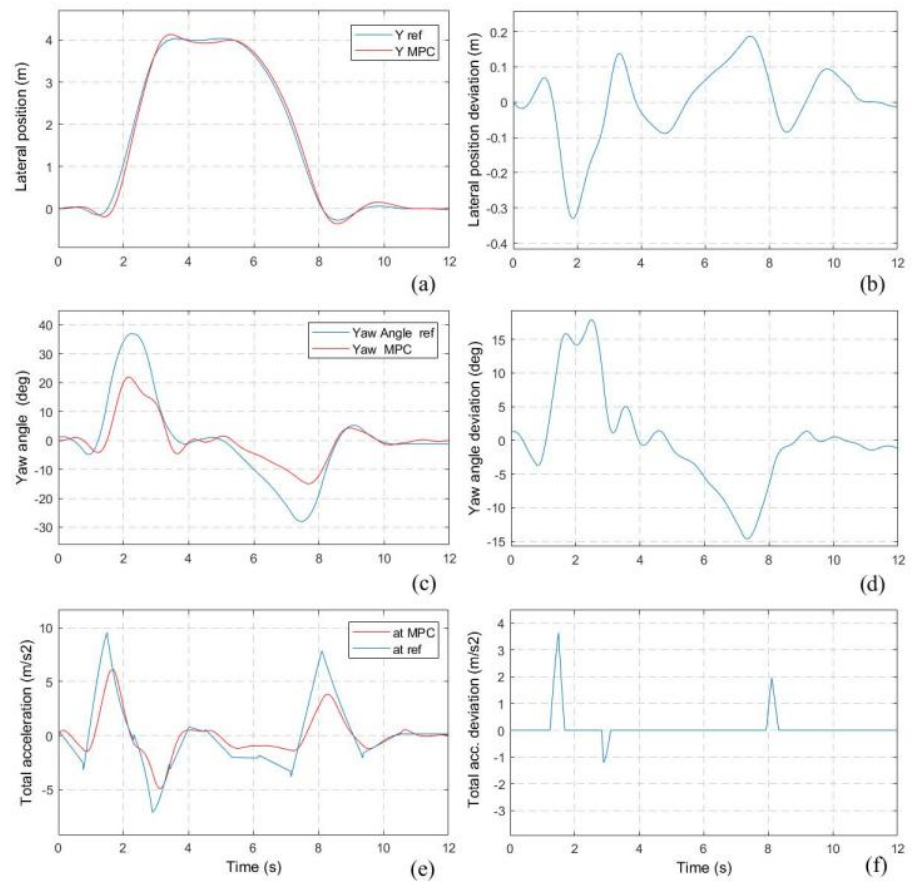

Figure 7: ISO3888-2 Moose test at $20 \mathrm{~km} / \mathrm{hr}$. MPC (a) Lateral position (b) Lateral position deviation (c) Yaw angle (d) Yaw angle deviation (e) Total acceleration (f) Total acceleration deviation

The controller manages to reduce the total acceleration for all three loss of control events below the maximum set value. This is done by changing the vehicle heading or yaw angle to a more stable direction while adhering to the limitations set on the available steering angle. The vehicle heading is changed by applying transitions of the steering angle at an acceptable rate with respect to the maximum value of steering angle and the maximum steering angle rate illustrated in Figure 8.

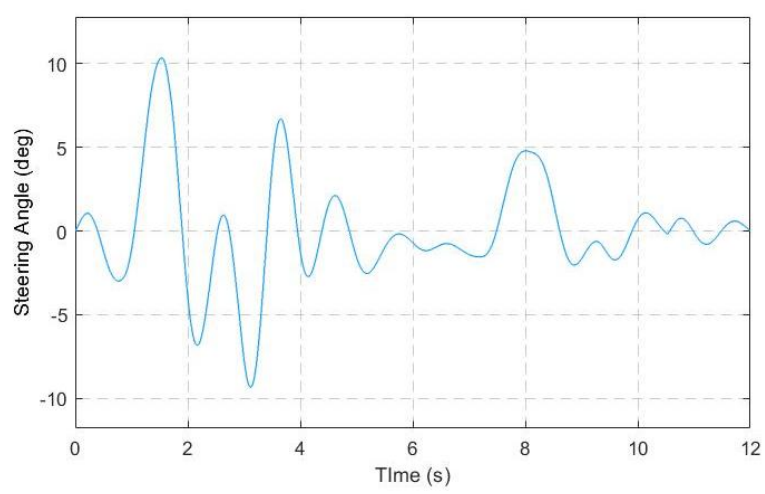

Figure 8: Steering angle required to navigate the moose test at $20 \mathrm{~km} / \mathrm{hr}$

The g-g diagram of the vehicle during the ISO3888 - 2 moose test shown in Figure 9 where the three events are presented. The total acceleration of the reference vehicle is outside the adhesion limit set by the friction circle which indicates the maximum acceleration of the vehicle. The controller reduces the maximum acceleration to be within the stability envelope of the friction circle.
Volume 4, Issue 5, pp 24-33, May-2020 https://doi.org/10.47001/IRJIET/2020.405005

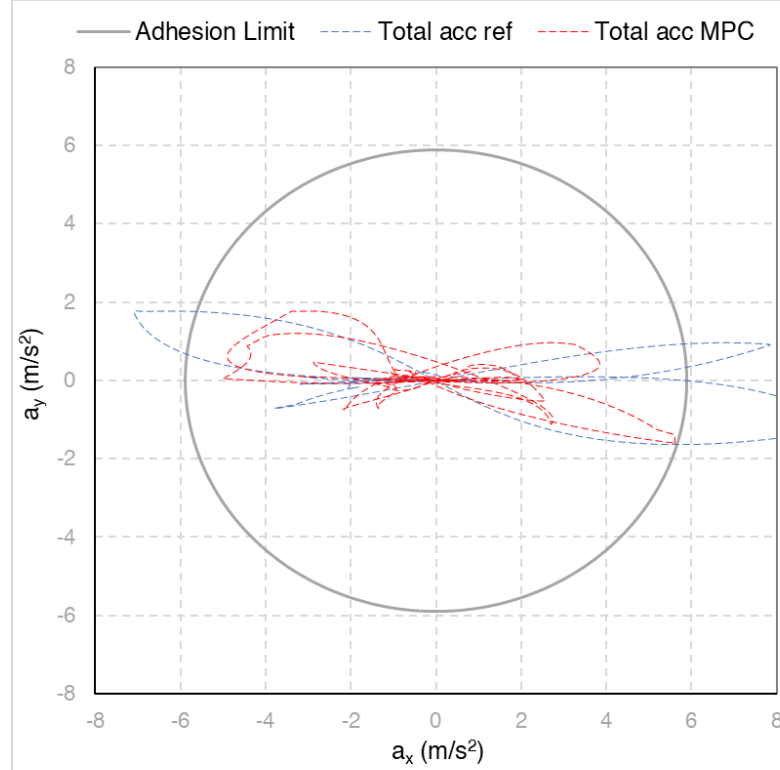

Figure 9: g-g diagram for ISO3888-2 Moose test at 20 km/hr

Figure 10, Error! Reference source not found. illustrate the vehicle response to the ISO3888-2 Moose test at $20 \mathrm{~km} /$ $\mathrm{hr}$ in terms of lateral velocity, slip angle, roll rate, roll angle, yaw rate and tire forces. The results also show a stable vehicle with no indication of loss of control or unacceptable behavior. The maximum slip angle of the vehicle is around 2.81deg and the maximum roll angle shown ins around $2.73 \mathrm{deg}$.
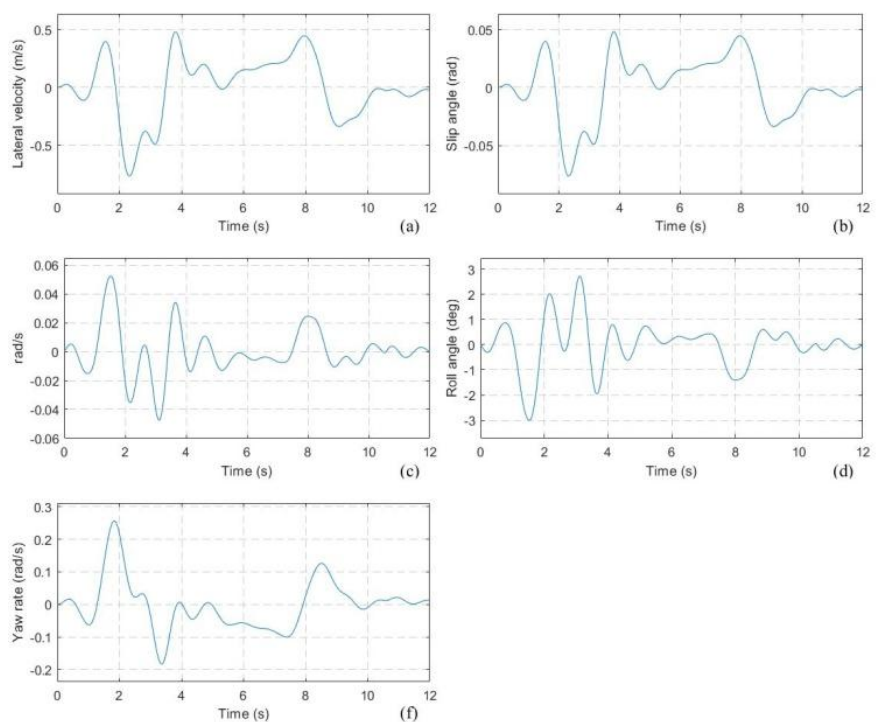

Figure 10: ISO3888-2 Moose test at $20 \mathrm{~km} / \mathrm{hr}$. vehicle response (a) Lateral velocity (b) Slip angle (c) Roll rate (d) Roll angle (e) Yaw rate

\section{b) Lane Change}

One of the most dangerous driving tasks is lane change maneuvers which result as the operator or autonomous system is required to avoid an obstacle at a high speed. The modeling and simulation of this maneuver is an essential step in the 
ISSN (online): 2581-3048

design of any autonomous system controller. The maneuver proposes high risk to the studied vehicle and other vehicle on the road. Therefore, the lane change maneuver was taken into consideration during the design and simulation process of the MPC. The proposed maneuver is illustrated in Figure 12 where the vehicle is driving at $36 \mathrm{~km} / \mathrm{hr}$ which is the maximum speed the vehicle can achieve. The MPC is introduced to an obstacle after $25 \mathrm{~m}$ and is required to change lanes at a lateral position of $3 \mathrm{~m}$.
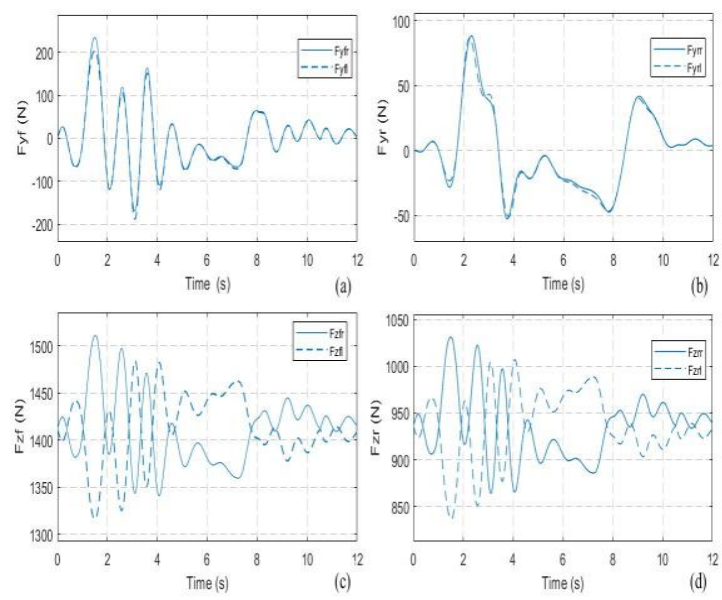

Figure 11: ISO3888-2 Moose test at $20 \mathrm{~km} / \mathrm{hr}$. Tire forces (a) Front tire side forces(b) Rear tire side forces (c) Front tire normal forces (d) Rear tire normal forces

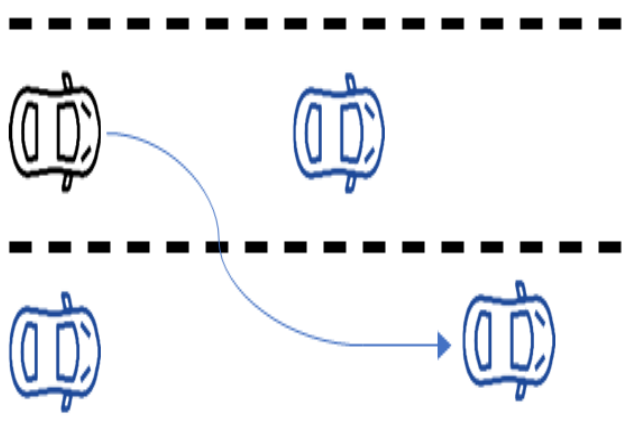

Figure 12: Simulated lane change maneuver

The dimensions and steps of the lane change maneuver is shown in Figure 13.

The simulation results are shown in Error! Reference source not found. toError! Reference source not found.. The MPC successfully maneuvers the lane change at maximum speed $36 \mathbf{k m} / \mathbf{h r}$ with minimal lateral position tracking error.

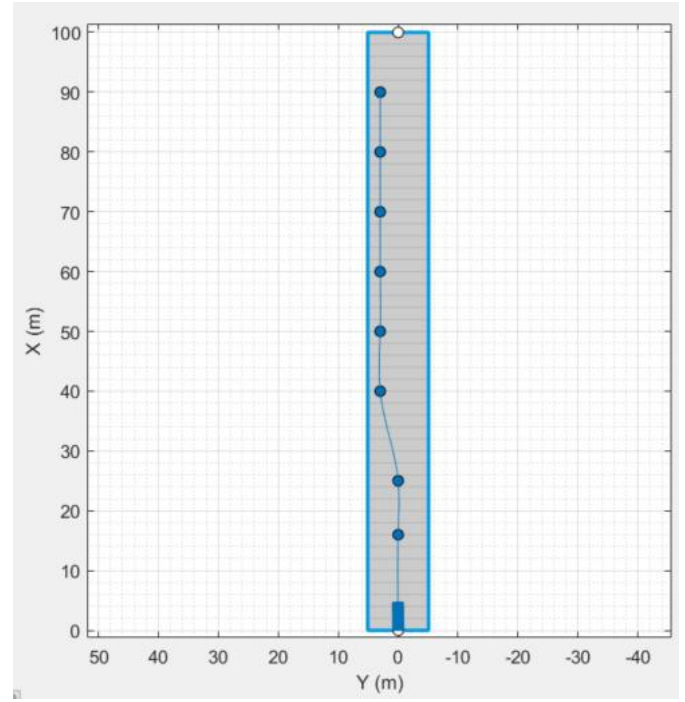

Figure 13: Simulated lane change maneuver in driving scenario designer

The maximum value of the lateral position deviation shown in Error! Reference source not found. (a), (b) is just more than $\mathbf{0 . 2 m}$ which occurs directly before the obstacle at $\mathbf{t}=\mathbf{2 . 8 3 \mathrm { s }}$ with total acceleration deviation of around $1.53 \mathrm{~m} / \mathrm{s}^{2}$. Deviation from the reference lateral position is also noticeable

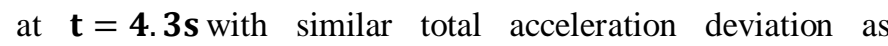
illustrated in Error! Reference source not found. (e), (f).
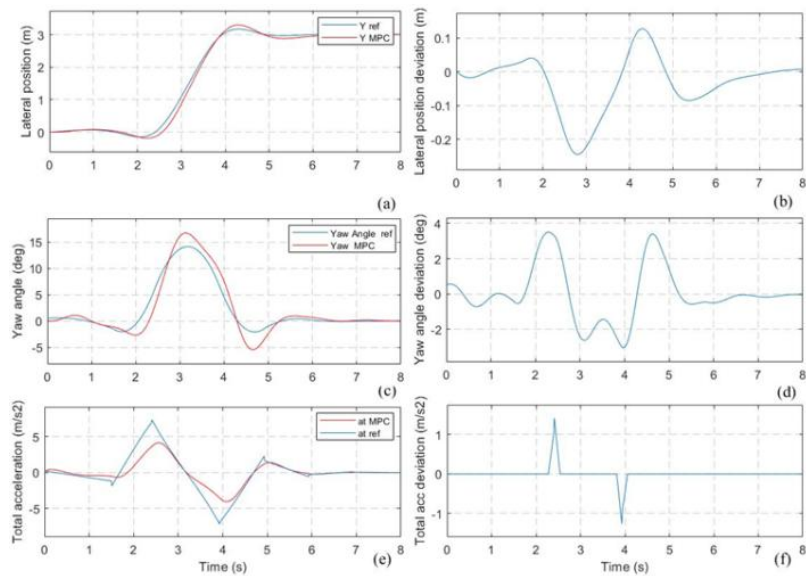

Figure 14: Lane change at $36 \mathrm{~km} / \mathrm{hr}$. MPC (a) Lateral position (b) Lateral position deviation (c) Yaw angle (d) Yaw angle deviation (e) Total acceleration (f) Total acceleration deviation

The proposed steering angle and the vehicle g-g diagram are shown in Error! Reference source not found. and Error! Reference source not found. while the vehicle response is shown in Error! Reference source not found. and Error! Reference source not found.. The result shown in the figures demonstrates the stability of the controller while executing the lane change maneuver even at maximum vehicle speed. 
ISSN (online): 2581-3048

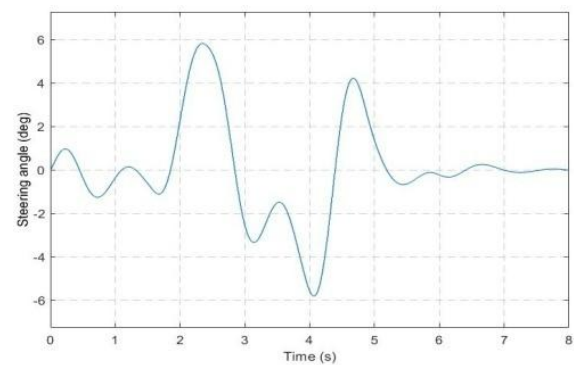

Figure 15: Steering angle required to navigate the lane change at 36 $\mathbf{k m} / \mathbf{h r}$

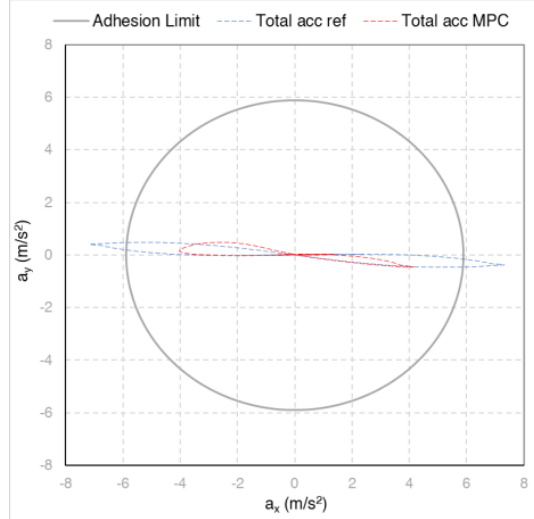

Figure 16: g-g diagram for lane change at $36 \mathrm{~km} / \mathrm{hr}$
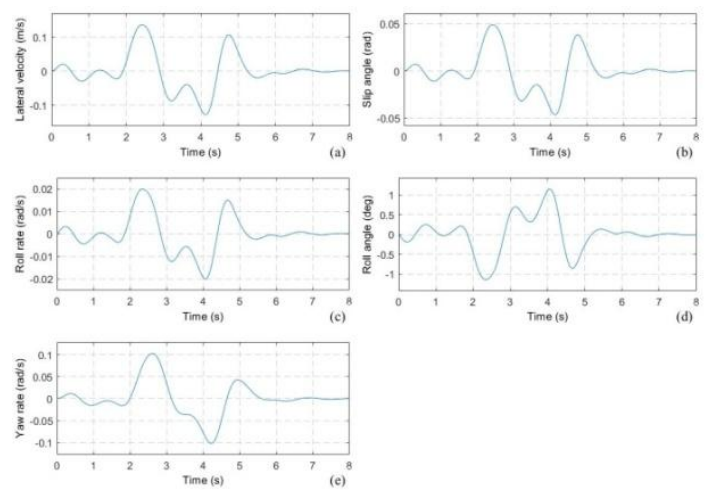

Figure 17: Lane change at $36 \mathrm{~km} / \mathrm{hr}$. vehicle response (a) Lateral velocity (b) Slip angle (c) Roll rate (d) Roll angle (e) Yaw rate
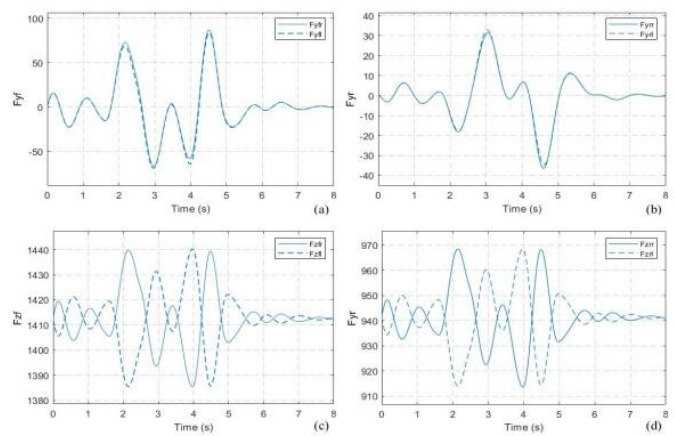

Figure 18: Lane change at $36 \mathrm{~km} / \mathrm{hr}$. Tire forces (a) Front tire side forces (b) Rear tire side forces (c) Front tire normal forces (d) Rear tire normal forces
https://doi.org/10.47001/IRJIET/2020.405005

\section{CONCLUSION}

In this research a controller for an SBW system was designed and simulated based on the Model predictive control theory for a small golf cart that is designed to operate autonomously. The controller was designed to accommodate the changes in loading conditions, vehicle properties and road conditions. An accurately controlled SBW system contributes in the optimization of driving strategies and the aids in the proper execution of vehicle maneuvers especially at the limits of handling. The model used in this research is the 4DOF one that includes yaw, lateral, longitudinal and roll motions which is more accurate and effective than the normally utilized bicycle model. Researches also consider the steering system dynamics as a constant gain which is not entirely accurate where in this research the steering system dynamics of the golf cart is taken into consideration. The model is coupled with a longitudinal forward dynamics model to calculate the longitudinal acceleration which is plotted against the lateral acceleration calculated from the lateral dynamics model. The total acceleration is then plotted on the g-g diagram of the vehicle against the friction circle obtained by the maximum total acceleration identified by braking tests. This is then used as limitations to the model predictive controller along with constraints on the steering wheel maximum angle and steering angle rate.

The target of the research was to develop an analytic model which is realistic and at the same time computationally efficient to be integrated in the MPC design. The model strikes a line between realism versus the linear bicycle model and computational efficiency versus more complicated several degrees of freedom model such as the 9 degrees of freedom model presented by Polack [6]. The designed MPC proves to be stable lower speeds and also at higher speeds in maneuvers close the vehicle limits. The controller manages to stabilize the vehicle even when the reference trajectory by the sensor data outputs high total acceleration outside the friction limit defined by the tire capabilities. The controller was simulated on two maneuvers which are the moose test (at different speeds) and a lane change. The controller was stable throughout the simulations giving excellent tracking performance in all simulated cases.

\section{FUTURE WORK}

The MPC designed in this research provided proper trajectory tracking at low and high speeds close to the vehicle handling limits which is acceptable but may be improved to reduce the tracking error to a minimal level. The suggestion to improve the performance of the controller includes: 
ISSN (online): 2581-3048

- Implementing a non-linear tire model which gives better insights on the vehicle handling characteristics which requires tire testing data provided by a tire testing machine. Where in this work the linear tire model was used due to the lack of availability of a tire testing machine.

- The use of actual sensor data provided by the finished autonomous golf car instead of relying on the data provided by the MATLAB driving scenario designer to optimize the controller performance.

- Adding the effect of pitch motion and weight transfer on each separate wheel which can be done using a more complicated 9 DOF model and using a separate g-g diagram for each wheel.

- The accuracy of the designed controller maybe also improved by designing a nonlinear MPC rather than the one used in this research. The nonlinear MPC can be more accurate especially If the tire model used was a nonlinear one. Where the linear MPC would fail to linearize the tire data. Although the nonlinear MPC requires high computational power, It gives higher accuracy during any maneuver.

- Coupling the lateral steering controller with a longitudinal controller to modulate the accelerator and brake pedals which gives more stable maneuvers by increasing or decreasing the longitudinal speed which directly affects the total acceleration.

- The implementation of the controller in the golf cart and experimental testing on the roads of the faculty of engineering Ain Shams University.

\section{REFERENCES}

[1] Borrelli, F., Falcone, P., Keviczky, T., Asgari, J., \&Hrovat, D. (2005). MPC-based approach to active steering for autonomous vehicle systems. International Journal of Vehicle Autonomous Systems, 3(2/3/4), 265. doi:10.1504/ijvas.2005.008237.

[2] Keviczky, T., Falcone, P., Borrelli, F., Asgari, J., \&Hrovat, D. (2006). Predictive control approach to autonomous vehicle steering. 2006 American Control Conference. doi:10.1109/acc.2006.1657458

[3] W. Khan, A. H. Abdullah, M. H. Anisi, and J. I. Bangash, "A comprehensive study of data collection schemes using mobile sinks in wireless sensor networks," Sensors, vol. 14, no. 2, pp. 2510-2548, 2014.

[4] Nitin R. Kapania, J. Christian Gerdes, Stanford.edu. (2014). An autonomous lanekeeping system for vehicle path tracking and stability at the limits of handling | Dynamic Design Lab.

[5] Kapania, N. R., \& Gerdes, J. C. (2015). Path tracking of highly dynamic autonomous vehicle trajectories via iterative learning control. 2015 American Control Conference (ACC). doi:10.1109/acc.2015.7171151.

[6] Funke, J., Brown, M., Erlien, S. M., \& Gerdes, J. C. (2017). Collision Avoidance and Stabilization for Autonomous Vehicles in Emergency Scenarios. IEEE Transactions on Control Systems Technology, 25(4), 1204-1216. doi:10.1109/tcst.2016.2599783.

[7] Polack, P., Altche, F., d' Andrea-Novel, B., \& de La Fortelle, A. (2017). The kinematic bicycle model: A consistent model for planning feasible trajectories for autonomous vehicles2017 IEEE Intelligent Vehicles Symposium (IV). doi:10.1109/ivs.2017.7995816.1

[8] Automotive Model Predictive Control Models, Methods and Applications, Del Re, L., Allgöwer, F., Glielmo, L., Guardiola, C., Kolmanovsky, I. (Eds.), ISBN 978-1-84996-071-7.

[9] Wang L. Model predictive control system design and implementation using MATLABß. Springer Science \& Business Media; 2009 Feb 14.

[10] Reza N. Jazar, "Vehicle Dynamics: Theory and Applications”, Springer, ISBN: 978-0-387-74243-4

[11] William F. Milliken, Douglas L. Milliken, "Race Car Vehicle Dynamics", SAE International, ISBN: 978-156091-526-3.

[12] ISO 3888-2: 2002, 2002. Passenger Cars-Test Track for a Severe Lane Change Manoeuvre-Part 2: Obstacle Avoidance.

\section{Citation of this Article:}

Mohamed Abdlshakour Allam, M.Ibrahim Abdelziz, Diaa Abidou, "Modeling, Simulation and Development of a Model Predictive Controller for an Autonomous Steer by Wire ADS-DV" Published in International Research Journal of Innovations in Engineering and Technology - IRJIET, Volume 4, Issue 5, pp 24-33, May 2020. https://doi.org/10.47001/IRJIET/2020.405005 